\title{
Bax activation and stress-induced apoptosis delayed by the accumulation of cholesterol in mitochondrial membranes
}

\author{
S Lucken-Ardjomande ${ }^{1}$, S Montessuit ${ }^{1}$ and J-C Martinou ${ }^{\star, 1}$
}

Activation of Bax or Bak is essential for the completion of many apoptotic programmes. Under cytotoxic conditions, these proteins undergo a series of conformational rearrangements that end up with their oligomerization. We found that unlike inactive monomeric Bax, active oligomerized Bax is partially resistant to trypsin digestion, providing a convenient read out to monitor Bax activation. Using this assay, we studied how the lipid composition of membranes affects tBid-induced Bax activation in vitro with pure liposomes. We report that Bax activation is inhibited by cholesterol and by decreases in membrane fluidity. This observation was further tested in vivo using the drug U18666A, which we found increases mitochondrial cholesterol levels. When incubated with tBid, mitochondria isolated from U18666A-treated cells showed a delay in the release of Smac/Diablo and Cytochrome $c$, as well as in Bax oligomerization. Moreover, pre-incubation with U18666A partially protected cells from stressinduced apoptosis. As many tumours display high mitochondrial cholesterol content, inefficient Bax oligomerization might contribute to their resistance to apoptosis-inducing agents.

Cell Death and Differentiation (2008) 15, 484-493; doi:10.1038/sj.cdd.4402280; published online 14 December 2007

Among the stimuli that lead to apoptosis, a series of stress conditions trigger the permeabilization of the mitochondrial outer membrane (MOM) and the release of pro-apoptotic factors that will activate caspases in the cytosol. This so-called intrinsic apoptotic pathway is tightly regulated by proteins of the Bcl-2 family, among which the pro-apoptotic members Bax and Bak are essential. They seem to be the central players whose activation results in MOM permeabilization.

Under resting conditions, whereas Bak essentially resides in the MOMs, Bax is found in loose association with membranes (mainly the MOMs) or as a soluble protein in the cytosol. Following many cytotoxic signals, Bax and Bak undergo a series of conformational rearrangements. ${ }^{1}$ Bax translocates to mitochondria and inserts into the MOMs in a form that cannot be detached by alkali treatment. Bax and Bak then uncover $\mathrm{N}$-terminal epitopes and oligomerize. Oligomerization, shown by size exclusion chromatography, ${ }^{2}$ crosslinking experiments ${ }^{3,4}$ and native gel electrophoresis, ${ }^{5}$ appears as the critical step resulting in MOM permeabilization. However, despite many efforts, it remains unclear how each of these conformational rearrangements is regulated, and how Bax/Bak oligomerization leads to the release of mitochondrial apoptogenic factors. Both processes are undoubtedly regulated by other members of the $\mathrm{Bcl}-2$ family, 6,7 but the participation of many unrelated proteins

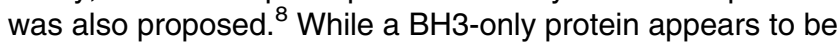
sufficient for Bax to oligomerize in synthetic liposomes, $, 9,10$ additional proteins could be required for its activation in isolated mitochondria. ${ }^{11}$ These might directly interact with Bax, and/or modify the membrane properties to allow its oligomerization. Interaction with mitochondrial lipids is indeed an important parameter to consider as the final conformational rearrangements that $\mathrm{Bax}$ and Bak have to undergo occur in a lipid bilayer. ${ }^{12}$

Recent studies started to underscore the fact that lipids play key roles in MOM permeabilization. ${ }^{1}$ Many groups immediately focused on cardiolipin, initially because it mediates the binding of Cytochrome $c$ to the mitochondrial inner membrane, and then again as it appears to be important for the activities of some members of the Bcl-2 family. ${ }^{13}$ Cardiolipin has often been shown to redistribute during apoptosis, and to be rapidly metabolized by oxidation and hydrolysis, which could be important for the release of mitochondrial apoptogenic factors. ${ }^{14-16}$ The influence of other lipids on the process of MOM permeabilization has so far been essentially studied in vitro by looking at the permeabilization of artificial liposomes by activated $\mathrm{Bax},{ }^{10,17}$ but probably because of difficulties associated with manipulating lipid levels in vivo, these studies were not pursued in cells. Moreover, an adequate Bax activation assay, allowing a reliable indication of the efficiency of its oligomerization, was missing. We developed a new method to detect oligomerized Bax, suitable to compare the degree of Bax oligomer formation under different conditions. Our in vitro studies show that Bax activation is inhibited by cholesterol and by decreases in membrane fluidity. These observations were confirmed in vivo, as treatment of HeLa

\footnotetext{
${ }^{1}$ Department of Cell Biology, University of Geneva, 30 quai Ernest-Ansermet, Geneva, Switzerland

*Corresponding author: J-C Martinou, Department of Cell Biology, University of Geneva, Sciences III, 30 quai Ernest-Ansermet, 1211 Geneva 4, Switzerland.

Tel: +4122 37964 43; Fax: + 412237964 42; E-mail: jean-claude.martinou@cellbio.unige.ch Keywords: apoptosis; bax; cholesterol; lipids; mitochondria

Abbreviations: MOM, mitochondrial outer membrane; MIM, mitochondrial inner membrane; PC, phosphatidylcholine; $\mathrm{CL}$, cardiolipin; PE, phosphatidylethanolamine; PI, phosphatidylinositol; CHO, cholesterol; DPH, 1,6-diphenyl-1,3,5-hexatriene; DOPC, dioleoyl-phosphatidylcholine; DSPC, distearoyl-phosphatidylcholine; FD20, FITC-dextran $20 \mathrm{kDa}$; U18666A, 3- $\beta$-[2-(diethylamino)ethoxy]androst-5-en-17-one; NPC1, Niemann-Pick Type C1

Received 04.7.07; revised 02.10.07; accepted 22.10.07; Edited by RJ Youle; published online 14.12.07
} 
cells with the drug U18666A led to the accumulation of cholesterol in mitochondrial membranes and delayed not only tBid-induced MOM permeabilization of isolated cholesterolenriched mitochondria, but also Bax oligomerization. In agreement with this, pre-incubation of HeLa cells with U18666A increased their resistance towards apoptosis-inducing agents.

\section{Results}

Oligomerized Bax is partially resistant to trypsin. Since Bax oligomerization has been directly associated with MOM permeabilization, the best way to assess Bax activation is by looking at its oligomerization. We have found that formation of Bax oligomers can be detected by analyzing the resistance of Bax to trypsin. As reported previously, when apoptosis was induced in HeLa cells by UV exposure, the level of Bax in mitochondria increased (Figure 1a). Mitochondria were then digested with trypsin and Bax was analyzed by immunoblotting. Bax could no longer be detected in control samples, whereas a trypsin-resistant Bax fragment (Tr-Bax) was found in samples from apoptotic cells. This Bax fragment (Tr-Bax) was not recognized by antibodies directed against the N-terminal end of Bax (data not shown), suggesting that the cleavage occurred between the epitopes recognized by the two different antibodies, probably at R34 or R37 of human Bax (Supplementary Figure 1). This is consistent with the conformation of activated Bax: cleavage of an exposed $\mathrm{N}$-terminal epitope,$^{18}$ and oligomerization, which would block access of trypsin to its potential cleavage sites. In support of this model, while untreated recombinant monomeric Bax was degraded by trypsin, Bax pre-incubated with octyl glucoside, which artificially triggers formation of Bax oligomers that can induce MOM permeabilization, ${ }^{19}$ was partially resistant to trypsin proteolysis (Figure 1b). In contrast, Bax unfolded and aggregated by heating was completely degraded by trypsin (Figure 1c). ${ }^{20}$ These observations suggest that partial resistance to trypsin is a characteristic feature of an active form of Bax oligomers.

Resistance to trypsin proteolysis can be used to study tBid-induced Bax oligomerization in vitro. Taking advantage of the resistance of oligomerized Bax to trypsin digestion, we set up an in vitro assay to examine the process of Bax activation induced by the BH3-only protein tBid. Synthetic MOM-like liposomes were incubated with recombinant Bax (His-tagged full-length Bax) in absence or presence of $\mathrm{tBid}$, and unbound proteins were removed by ultracentrifugation. The fraction of Bax bound to liposomes a

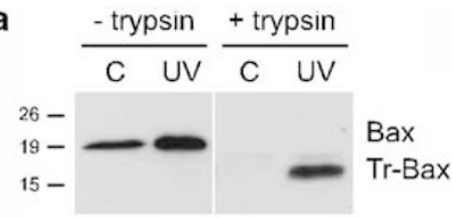

b

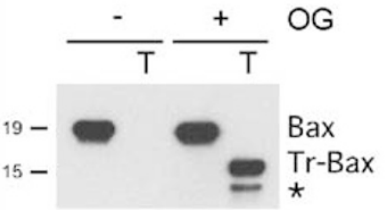

C

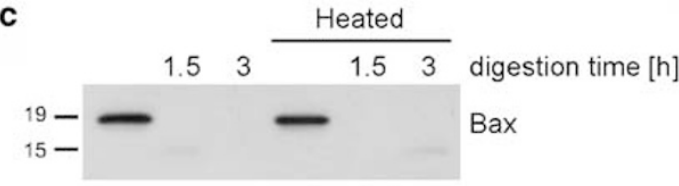

d

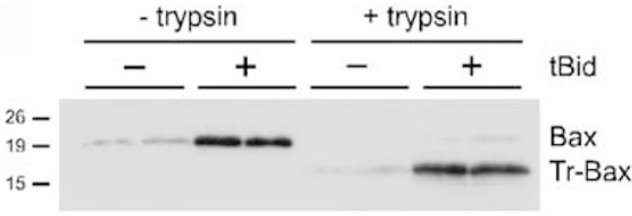

e

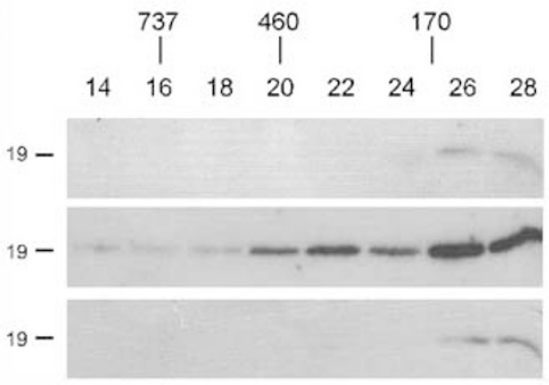

62

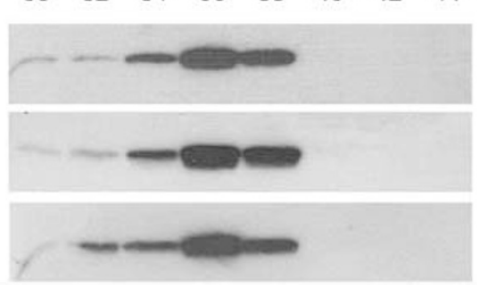

30
$\mathrm{kDa}$

Fractions

।

II

III

Figure 1 Oligomerized Bax is partially resistant to trypsin degradation. (a) Mitochondria of HeLa cells, untreated (C) or 16h after UV irradiation (UV), were isolated and digested with trypsin. Endogenous Bax was analyzed by immunoblotting. A trypsin-resistant Bax fragment (Tr-Bax) was only detected in mitochondria purified from apoptotic cells. (b) Recombinant Bax was incubated with or without $2 \%$ octyl glucoside (OG) and digested with trypsin (T). Bax was analyzed by immunoblotting. Only OG-treated Bax showed a partial resistance to trypsin digestion, with formation of a main $15 \mathrm{kDa}$ Tr-Bax fragment and of a smaller fragment $\left({ }^{*}\right)$, which probably appeared because, in the presence of a detergent, additional trypsin cleavage sites were accessible in the oligomers. Blot is representative of two independent experiments. (c) Recombinant Bax was heated for $20 \mathrm{~min}$ at $90^{\circ} \mathrm{C}$, cooled down on ice, then digested with trypsin for 1.5 or $3 \mathrm{~h}$, and analyzed by immunoblotting. (d) MOM-like liposomes (PC/CL/PE/PI/CHO 48/6/25/ 12/9) were incubated with recombinant Bax (100 nM) in presence or in absence ( + and -$)$ of tBid $(10 \mathrm{nM})$, and pelleted by ultracentrifugation. The amount of Bax bound to liposomes and the fraction resisting to subsequent trypsin proteolysis were analyzed by immunoblotting. Results show duplicate experiments. (e) Liposomes (I and II: PC/CL 60/40; III: PC/CL/CHO 40/40/20) were incubated with Bax (I) or Bax and tBid (II and III). Proteins were solubilized with CHAPS, separated by size exclusion chromatography, and the elution profile of Bax was analyzed by immunoblotting. For each condition, even-numbered fractions were loaded on two separate gels; a short exposure time was chosen for the low molecular weight fractions (30-44), and a longer one for the high molecular weight fractions (14-28) 
and its sensitivity to trypsin digestion were analyzed by immunoblotting. The presence of tBid was required for the recruitment of Bax to liposomes and led to the appearance of a Tr-Bax fragment (Figure 1d). Similarly to the permeabilization of membranes induced by tBid-activated Bax, 9,21 Tr-Bax formation was dependent on the concentration of tBid, and did not occur upon incubation with a mutant tBid, tBidmIII-2 (IGDE $\rightarrow$ AAAA), which has an intact membranebinding region but no functional $\mathrm{BH} 3$ domain (Supplementary Figure $2 a$ and $b$ ). In addition, similarly to the inhibition of Bax insertion and oligomerization by antiapoptotic members of the Bcl-2 family, ${ }^{4,9}$ Tr-Bax formation was inhibited by recombinant His-tagged $\mathrm{Bcl}-\mathrm{x}_{\mathrm{L}}$ lacking its C-terminal tail (Supplementary Figure $2 \mathrm{c}$ ). Bcl- $\mathrm{x}_{\mathrm{L}}$ also prevented the association of $\mathrm{tBid}$ with liposomes, perhaps sequestering it in a soluble complex. This could be specific to the recombinant Bcl- $x_{L}$ used, which, lacking its $\mathrm{C}$-terminal helix, has a free hydrophobic pocket that may be occupied by the $\mathrm{BH} 3$ motif of tBid. Extensive digestion of the samples (either by using a higher trypsin concentration or with a longer incubation time) led to the disappearance of the Tr-Bax band (Supplementary Figure $3 a$ and $b$ ), suggesting that Tr-Bax represents a stabilized digestion intermediate, but can still be further degraded by trypsin.

To confirm that the presence of Tr-Bax was illustrative of Bax oligomerization, we incubated liposomes with Bax and tBid, and analyzed the distribution of Bax by size exclusion chromatography and immunoblotting. In the absence of tBid, Bax eluted as a monomer (Figure 1e, line I). However, in the presence of $\mathrm{tBid}$, a significant amount of Bax shifted to high molecular weight fractions (Figure 1e, line II), indicating formation of oligomers. Trypsin digestion of the fractions confirmed that only oligomeric Bax was resistant to trypsin degradation (Supplementary Figure $4 \mathrm{a}$ and b); conversely, analysis of Tr-Bax by size exclusion chromatography confirmed that it only eluted in high molecular weight fractions (Supplementary Figure 4c). Partial trypsin digestion was directly linked to the quaternary structure of Bax and did not simply result from a protection by the bilayer in which Bax was embedded, as Tr-Bax was still produced if trypsin digestion was performed on proteins that had been extracted from liposomes with CHAPS (Supplementary Figure 5). In the presence of CHAPS however, a smaller Tr-Bax fragment was finally obtained, suggesting, as previously observed with $O G$ (Figure 1b), that detergents slightly unfold the oligomer, making additional sites more accessible to trypsin.

Collectively, these experiments show that the amount of Tr-Bax formed is representative of the efficiency of Bax oligomerization. This in vitro assay thus represented a suitable tool to study how the characteristics of liposomes affect the process of tBid-induced Bax activation.

\section{Cholesterol and membrane order inhibit Bax activation} in vitro. Similar to the result described above with MOM-like liposomes, tBid promoted the oligomerization of Bax in simple phosphatidylcholine (PC)/cardiolipin (CL) mixtures (Figure 2a). Addition of cholesterol decreased the amount of Bax resistant to trypsin digestion, both using high (Figure 2a) and low (Supplementary Figure 6) CL-containing liposomes. Size exclusion chromatography confirmed that the presence of cholesterol diminished the amount of Bax found in high molecular weight fractions (Figure 1e, lines II and III). Interestingly, cholesterol decreased neither the amount of Bax bound to liposomes (Figure 2a and Supplementary Figure 6) nor its degree of insertion, as the fraction of Bax remaining in membranes after an alkali treatment even increased slightly when cholesterol was included in the assay (Figure $2 b$ ). Similarly, the presence of cholesterol appeared to promote the binding of tBid to liposomes (Figure 2a), and also to slightly potentiate the binding of recombinant Fis (Figure 2c), a mitochondrial protein with a single transmembrane domain and which can insert in the MOMs of isolated mitochondria (data not shown). While Bax displayed decreased resistance to trypsin digestion in presence of cholesterol, Fis was completely degraded using both types of liposomes, showing that cholesterol did not directly impact on the activity of trypsin (Figure 2c).

Activated Bax was previously shown to provoke membrane permeabilization of synthetic liposomes, allowing the release of large dextrans contained within them. ${ }^{9,10}$ We therefore entrapped $20 \mathrm{kDa}$ FITC-labeled dextrans (FD20) in liposomes, and measured the amount of FD20 released in the supernatants upon incubation with Bax, tBid or a combination of the two (Figure 2d). Bax and tBid were both required for the efficient permeabilization of liposomes. In agreement with its effect on Bax oligomerization, cholesterol significantly inhibited FD20 release. Together, these data suggest that cholesterol specifically hinders the process of Bax oligomerization, impacting on membrane permeabilization. As cholesterol did not inhibit the insertion of Bax in bilayers, and as this process requires tBid, it seems unlikely that cholesterol simply prevented the interaction between the two proteins.

We then used steady state fluorescence anisotropy to look at the effects of cholesterol on the fluidity of such liposomes. ${ }^{22}$ At $20 \%$, cholesterol significantly increased the anisotropy of the fluorescent hydrophobic probe 1,6-diphenyl-1,3,5-hexatriene (DPH), indicating a decrease in the fluidity of the membrane (Figure 2e). Cholesterol is a well-known regulator of membrane fluidity, as is the degree of unsaturation of the fatty acyl chains of phospholipids. While saturated fatty acyl chains can be tightly packed together, unsaturations create kinks, decreasing lateral packing and membrane order. Natural lipids used for the experiments described above contained a majority of mono- and di-unsaturated fatty acyl chains. Similarly, liposomes composed of mono-unsaturated dioleoyl-phosphatidylcholine (DOPC, C18:1), and beef heart cardiolipin (essentially substituted with di-unsaturated linoleates, C18:2) sustained tBid-induced Bax binding and oligomerization (Figure 2f). Gradually replacing DOPC with saturated distearoyl-phosphatidylcholine (DSPC, C18:0) diminished the amount of Tr-Bax formed, while leaving the binding of Bax and tBid essentially unchanged (Figure 2f). Like cholesterol, DSPC increased the anisotropy of DPH (Figure 2e), consistent with the fact that a decrease in membrane fluidity prevents Bax oligomerization.

Benzyl alcohol is an anaesthetic that increases the fluidity of lipid bilayers. While it did not have a significant impact on the fluorescence anisotropy of DPH in PC/CL 60/40 liposomes, it significantly reduced it in $\mathrm{PC} / \mathrm{CL} / \mathrm{CHO} 40 / 40 / 20$ liposomes (Figure 2e) and increased the efficiency of Bax oligomerization (Figure 2g). BA also inhibited the binding of tBid, but the 


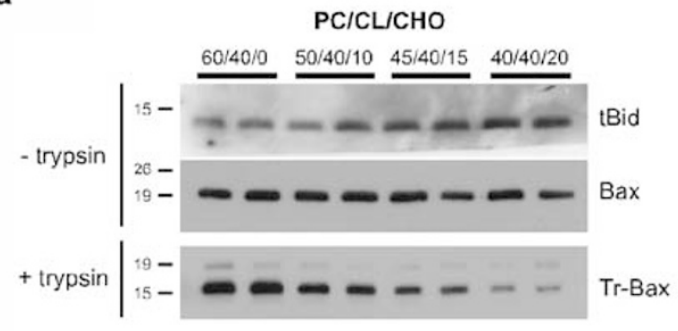

d

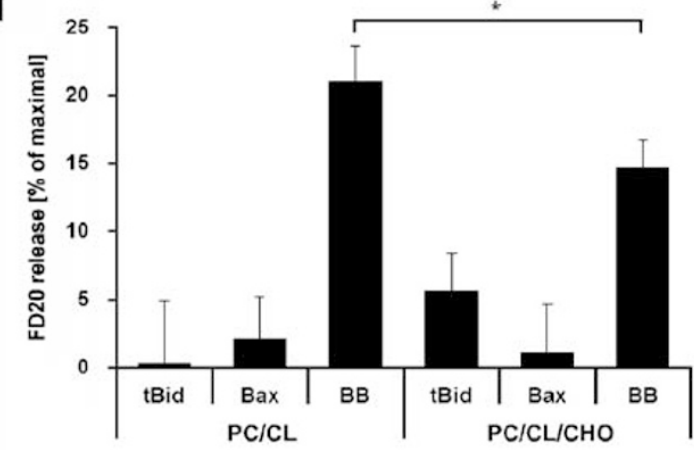

f

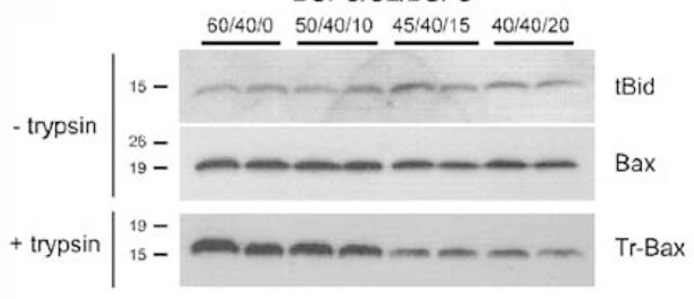

b

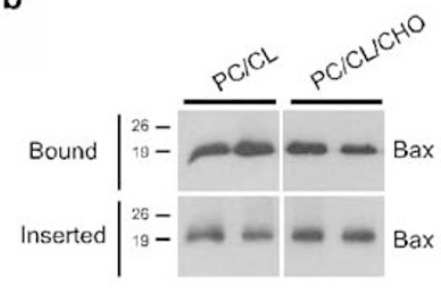

C
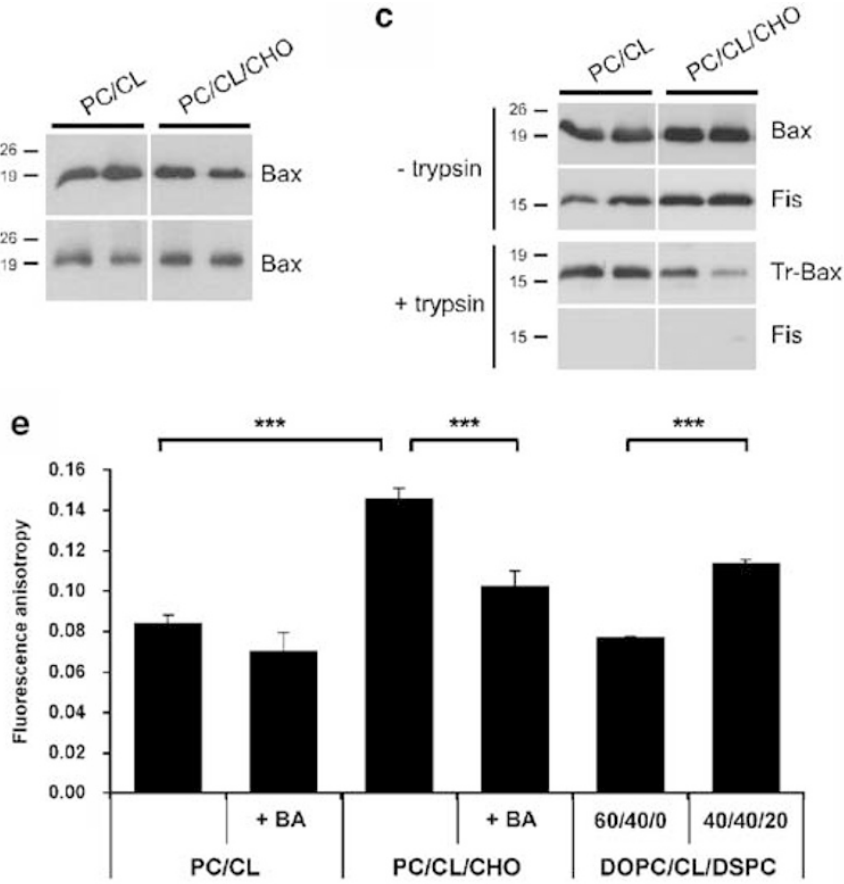

g

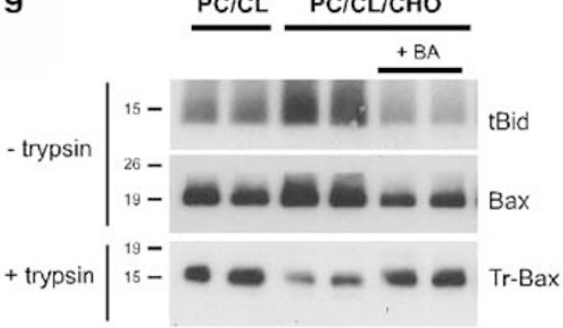

Figure 2 tBid-induced Bax oligomerization in vitro is inhibited by decreases in membrane fluidity. Liposomes containing PC, CL and increasing amounts of cholesterol (a) or DSPC (f) were incubated with Bax (100 nM) and tBid $(10 \mathrm{nM})$, and ultracentrifuged. Pellets were subjected to immunoblotting to analyze the binding of Bax and of tBid to the liposomes ( - trypsin), as well as the fraction of Bax resistant to a subsequent trypsin digestion step ( + trypsin). (b) PC/CL 60/40 and PC/CL/CHO 40/40/20 liposomes were incubated with Bax and tBid, ultracentrifuged, and loosely bound proteins were removed by alkali extraction and centrifugation. Bax inserted in the membranes was analyzed by immunoblotting the pellet. (c) PC/CL 60/40 and PC/CL/CHO 40/40/20 liposomes were incubated with recombinant Fis (50 nM), Bax and tBid, and ultracentrifuged. The amounts of Fis and of Bax associated with liposomes were determined by immunoblotting the pellets (- trypsin), and the fractions of Bax and of Fis remaining after an incubation with trypsin were assayed ( + trypsin). In (b and $\mathbf{c}$ ) results for PC/CL and PC/CL/CHO were parts of the same blots and corresponded to identical exposure times. All the blots show duplicates and are representative of at least three independent experiments. (d) FD20 release assays were performed with PC/CL 60/40 and PC/CL/CHO 40/40/20 liposomes. The fraction of FD20 released in the supernatants recovered after incubation with tBid, Bax, or tBid and Bax (BB) was calculated relative to maximal release obtained with $1 \%$ Triton. Values are means of five independent experiments \pm S.E.M. ${ }^{*} P<0.04$. (e) $P C / C L 60 / 40$ and $P C / C L / C H O ~ 40 / 40 / 20$, without or with benzyl alcohol (BA, $50 \mathrm{mM}$ ), DOPC/CL 60/40, or DOPC/CL/DSPC 40/40/20 liposomes were incubated with the lipophilic probe DPH and its steady-state fluorescence anisotropy was measured. Values are the average of three or six experiments \pm S.E.M. ${ }^{* \star *} P<0.001$. (g and $\left.\mathbf{h}\right) \mathrm{BA}(50 \mathrm{mM})$ was added to PC/CL/CHO $40 / 40 / 20(\mathbf{g})$ or to PC/CL $60 / 40$ liposomes (h) and Bax activation assays were performed as described previously. Blots are representative of three independent experiments and in (g), show duplicates

level reached was sufficient to trigger maximal Bax binding. This point is supported by the dose response curve in Supplementary Figure $2 \mathrm{a}$. Therefore, increasing the fluidity of the bilayer of $\mathrm{PC} / \mathrm{CL} / \mathrm{CHO}$ liposomes restores efficient Bax oligomerization.

Collectively, these experiments suggest that decreasing the fluidity of membranes appears to specifically reduce tBidinduced Bax oligomerization without inhibiting Bax insertion into liposomes.

Treatment of HeLa cells with U18666A leads to the accumulation of cholesterol in mitochondria. To validate our in vitro data in vivo, it was first essential to modulate mitochondrial cholesterol levels. U18666A is a drug that induces intracellular cholesterol accumulation, an abnormality that is encountered in Niemann-Pick Type C1 disease. It interferes with the trafficking of cholesterol, causing its increase in late endosomes/lysosomes, ${ }^{23,24}$ and inhibits its neosynthesis in the ER. ${ }^{25} \mathrm{~A}$ recent study reported the accumulation of cholesterol in the mitochondria of neurons isolated from an Niemann-Pick Type $\mathrm{C} 1$ mouse model, ${ }^{26}$ raising the possibility that U18666A might also increase mitochondrial cholesterol content. We therefore incubated HeLa cells with U18666A, isolated mitochondria, and analyzed their lipid composition by thin-layer chromatography (Figure $3 a$ and $b$ ). The level of free cholesterol was increased by about $25 \%$, leaving the amounts 
of the major phospholipids essentially unchanged. We also used an assay based on the quantification of $\mathrm{H}_{2} \mathrm{O}_{2}$ formed upon cholesterol oxidation and observed a rise in the total cholesterol content of mitochondria isolated from U18666Atreated cells by about $50 \%$ (Figure $3 \mathrm{c}$ ). The difference between the two results is partly explained by the contribution of esterified cholesterol to the latter quantification.

Formation of a lighter buoyant fraction of lysosomes was reported when fibroblasts were incubated for over a day with U18666A. ${ }^{23}$ We therefore ran crude mitochondrial fractions on continuous $8-48 \%$ sucrose gradients and analyzed the distribution of mitochondrial proteins by immunoblotting (Figure 3d). Mitochondria isolated from control cells were mainly found in pellets, but those isolated from U18666Atreated cells were more concentrated in fractions corresponding to $48-43 \%$ sucrose, indicating that incubation with U18666A led to a significant decrease in the density of mitochondria. In compensation for this change, the proportion of other heavy organelles such as peroxysomes increased in the pellet, as shown by immunoblotting with an antibody directed against Catalase (Figure 3d). Treatment with U18666A did not, however, induce any gross change in mitochondrial morphology (Figure 3e), nor did it alter the expression levels of various mitochondrial proteins (Figure 3f). Moreover, the degree of contamination of crude mitochondrial preparations by various intracellular organelles was similar when prepared from untreated or from U18666A-treated cells (Supplementary Figure 7).
Together, these observations show that pre-incubation of HeLa cells with U18666A is an adequate way to increase mitochondrial cholesterol content. Notably, U18666A exposure did not affect processes that are known to modulate the sensitivity of cells to apoptosis, such as the morphology of mitochondria or the expression levels of pro- and anti-apoptotic proteins.

\section{Cholesterol-enriched mitochondria are less sensitive} to tBid-induced MOM permeabilization. Addition of tBid to isolated mitochondria was previously shown to be sufficient to activate endogenous Bax and to trigger MOM permeabilization. ${ }^{4,21}$ We isolated crude mitochondria from control and U18666A-treated HeLa cells and incubated them with recombinant $\mathrm{tBid}$. Smac/Diablo and Cytochrome $c$ release from cholesterol-enriched mitochondria were delayed (Figure 4a). We assayed Bax activation initially by looking at the amount of Bax remaining in a membrane pellet after incubating the mitochondria in an alkaline carbonate solution, and then by testing its resistance to trypsin. Bax insertion was more efficient in cholesterol-enriched mitochondria (Figure 4b). However, the formation of $\mathrm{Tr}$-Bax was delayed in mitochondria isolated from U18666A-treated cells (Figure 4c), suggesting that Bax oligomerization is specifically slowed down by a rise in the cholesterol content of mitochondria.

Recent reports indicated that U18666A might have direct effects on the properties of membranes. ${ }^{27}$ We therefore repeated this in vitro assay with control mitochondria that had a
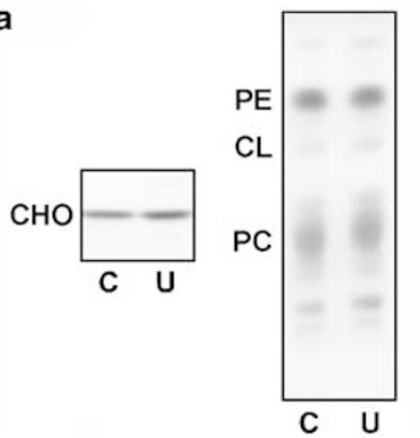

b

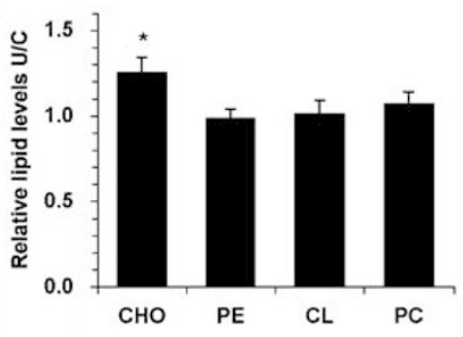

e

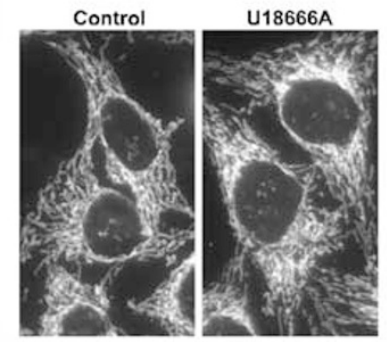

C
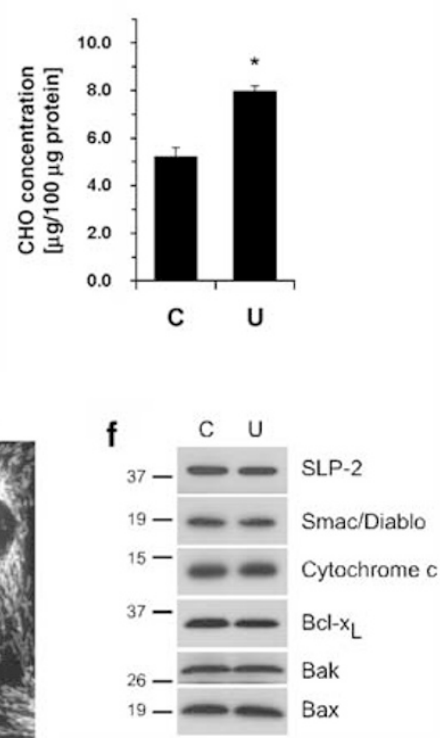

Figure 3 U18666A induces an accumulation of cholesterol in the mitochondria of HeLa cells. HeLa cells were incubated with (U) or without (C) U18666A. (a) Analysis of the lipid composition of isolated crude mitochondria by thin-layer chromatography. (b) The intensities of the spots corresponding to CHO, PC, CL and PE were quantified by image analysis, and $\mathrm{U} / \mathrm{C}$ ratios were calculated. Values represent means of four independent experiments \pm S.E.M. ${ }^{*} P<0.03$. (c) Total cholesterol levels of crude mitochondria were quantified using an assay based on the production of $\mathrm{H}_{2} \mathrm{O}_{2}$ upon cholesterol oxidation. Cholesterol concentrations $(\mu \mathrm{g} / 100 \mu \mathrm{g}$ protein) are displayed and represent an average of three independent experiments. ${ }^{*} P<0.02$. (d) Mitochondria isolated from HeLa cells were run on continuous $8-48 \%$ sucrose gradients. The pellets and two fractions corresponding to $48-43 \%$ sucrose and $43-38 \%$ sucrose were analyzed by immunoblotting, using antibodies directed against three mitochondrial proteins (SLP-2, Smac/Diablo and Cytochrome c) and a peroxisomal protein (Catalase). Equal loading was checked by staining the membrane with Coomassie blue. The blot is representative of three independent experiments. (e) HeLa cells were immunostained with an antibody directed against Cytochrome $c$ to observe the morphology of their mitochondria. (f) The levels of expression of various mitochondrial proteins were analyzed by immunoblotting isolated crude mitochondria 
a

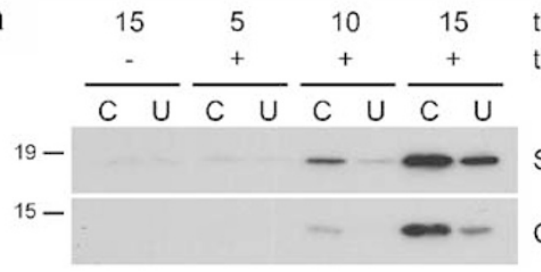

b

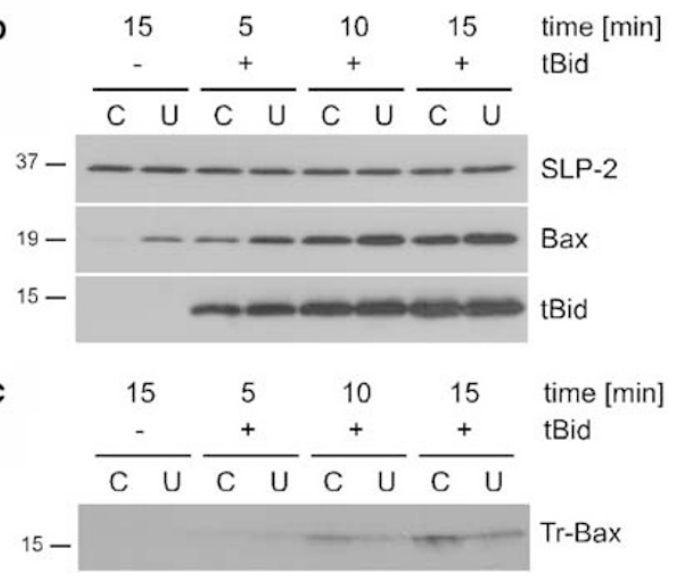

Figure 4 tBid-induced MOM permeabilization and Bax oligomerization are delayed in mitochondria isolated from U18666A-treated cells relative to control cells. Crude mitochondria isolated from control (C) or U18666A-treated cells (U) were left untreated or incubated with tBid for 5,10 , and $15 \mathrm{~min}$, and recovered by centrifugation. (a) The amounts of Smac/Diablo and Cytochrome $c$ released in the supernatants were assessed by immunoblotting. (b) The mitochondrial pellets were further treated with an alkaline solution of sodium carbonate, ultracentrifuged, and the pellets were analyzed by immunoblotting to look at the insertion of Bax and tBid in the membranes. SLP-2 was used as a loading control. (c) Alternatively, the mitochondrial pellets were digested with trypsin and the resistance of Bax to proteolysis was analyzed by immunoblotting. Blots are representative of at least four independent experiments

been pre-incubated with various concentrations of U18666A. This treatment did not delay tBid-induced Smac/Diablo and Cytochrome $c$ release (Supplementary Figure 8).

Collectively, these observations indicate that, in agreement with data obtained with synthetic liposomes, accumulation of cholesterol in mitochondrial membranes inhibits Bax oligomerization. Moreover, they support a Bax activation model in which Bax insertion is not necessarily accompanied by its oligomerization and MOM permeabilization. ${ }^{28}$

HeLa cells treated with U18666A are less susceptible to apoptosis-inducing agents. To test if an accumulation of cholesterol in mitochondria also modulates the sensitivity of cells to cytotoxic agents, HeLa cells treated with U18666A were incubated with actinomycin $D$ and were fractionated at different time points. In agreement with data obtained in vitro, Cytochrome $c$ and Smac/Diablo release from mitochondria were delayed in U18666A-treated cells in comparison to control cells (Figure $5 a$ and $b$ ). This delay was also clearly seen in the pattern of activation of caspase-3 (Figure 5c). When looking at Bax activation, its insertion in the MOM was similar under both conditions and at all times tested (Figure 5d), but it became resistant to trypsin degradation more quickly in control cells (Figure 5e), further supporting data obtained in vitro.
To quantify the protection provided by U18666A, we then treated cells with two apoptotic stimuli, actinomycin $D$ or staurosporine, and assayed apoptosis by Annexin-V staining and FACS analysis. Pre-incubation of cells with U18666A significantly delayed Annexin-V labelling (Figure 5f). This protection was however only moderate, probably because U18666A perturbs cholesterol homoeostasis at multiple levels and has side effects deleterious for cells.

The protection provided by treatment with U18666A was specific to mitochondria-dependent apoptotic stimuli. Indeed, U18666A rather increased the sensitivity of 293T cells to FasL, which promotes cell death independently of mitochondria (Supplementary Figure 9).

Together, these data show that treatment of HeLa cells with U18666A increases their resistance to apoptosis-inducing agents, partly because of an inhibition of Bax oligomerization.

\section{Discussion}

In this article, we describe a new method to assess Bax oligomerization, based on the observation that unlike monomeric Bax, oligomeric Bax is partially resistant to trypsin degradation. This allowed us to study in vitro how the composition of liposomes influences Bax oligomer formation, and to dissociate Bax oligomerization from its insertion in lipid bilayers. A similar assay could be developed for Bak, and would help to better understand its activation process. However, difficulties in purifying full-length Bak would require tests to be performed with in vitro translated Bak, or in isolated mitochondria with the endogenous protein.

We observed that Bax activation is modulated by the fluidity of the lipid bilayer and that increasing the cholesterol/ phospholipids mass ratio to $25 \%$, significantly inhibits tBidinduced Bax oligomerization and decreases the membrane permeabilization that follows. Cholesterol prevents Bax activation after its insertion into the MOMs, but prior to its oligomerization, and does not inhibit tBid-induced recognition of Bax by $\mathrm{N}$-terminus-specific antibodies (data not shown). These observations support the notion that Bax activation can be regulated late in the course of its sequential conformational rearrangements. ${ }^{5,28}$ Membrane order might in fact stabilize monomeric Bax in one of its intermediate conformations. Following exposure to cytotoxic agents, Bax was shown to insert in membranes with at least three transmembrane helices. ${ }^{28}$ However, it remains unclear if they insert simultaneously or if the C-terminal helix precedes the others. It would be interesting to use the mutant Bax proteins described by Annis et al., ${ }^{28}$ and to test if any insertion events are affected by cholesterol.

In mammalian MOMs, the cholesterol/phospholipid mass ratio was reported to be between 3 and $24 \%{ }^{29}$ In the plasma membrane, cholesterol has the well-known capacity to induce formation of ordered microdomains, characterized by their resistance to detergent-induced solubilization. Some mitochondrial proteins were recently reported to remain insoluble under similar conditions, suggesting that analogous lipid rafts might be present in mitochondrial membranes. ${ }^{30}$ If such microdomains exist in the MOMs, our results suggest that membrane order might locally impede Bax oligomerization. However, treatment of mitochondria isolated from human 
a

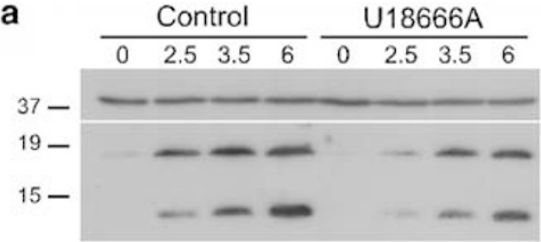

b

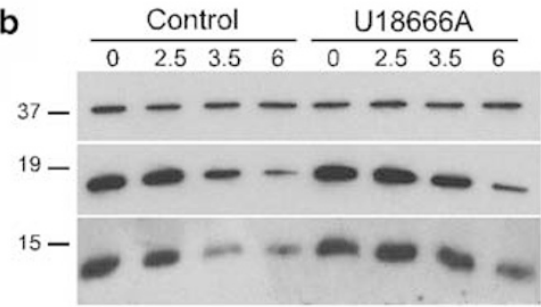

C

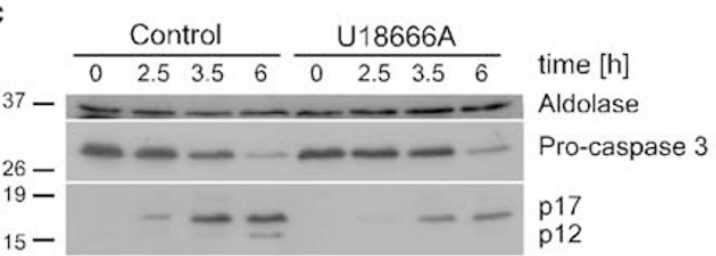

d

time [h]

Aldolase

Smac/Diablo

Cytochrome c

time $[\mathrm{h}]$

SLP-2

Smac/Diablo

Cytochrome c

p12

e
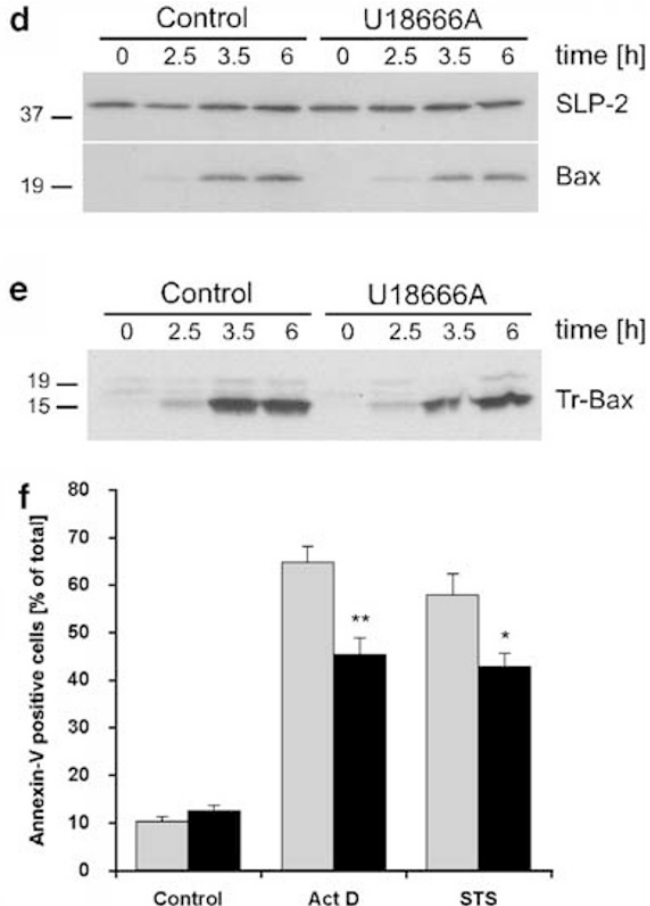

Figure 5 HeLa cells treated with U18666A are more resistant to stress-induced apoptosis than control cells. HeLa cells were incubated with U18666A for 2 days, left untreated, or incubated with actinomycin $D$ for $2.5,3.5$, or $6 \mathrm{~h}$. Cells were then fractionated and analyzed by immunoblotting. Blots are representative of at least five independent experiments. The levels of Smac/Diablo and Cytochrome $c$ were assessed in the cytosolic (a) and mitochondrial fractions (b). Aldolase and SLP-2 were respectively used as loading controls. (c) Caspase-3 activation was analyzed in the cytosolic fractions with an antibody that detects pro-caspase-3 and its two active fragments, p17 and p12. The amount of Bax inserted in the mitochondrial membranes was revealed by analyzing its presence in the pellets obtained after a sodium carbonate treatment of the mitochondria (d), and the amount of oligomerized Bax was revealed after treating the mitochondria with trypsin (e). (f) Apoptosis was examined in control (grey bars) or U18666A-treated (black bars) HeLa cells incubated with actinomycin D (Act D, 6 h) or with staurosporine (STS, $6 \mathrm{~h}$ ), by Annexin-V staining and FACS analysis. The percentage of Annexin-V-positive cells is shown. Values are the average of at least five independent experiments, \pm S.E.M. ${ }^{*} P<0.02,{ }^{* \star} P<0.004$

lymphoblastoid cells, with the cholesterol chelator methyl$\beta$-cyclodextrin ( $\mathrm{M} \beta \mathrm{c}, 500 \mu \mathrm{g} / \mathrm{ml}$ ) was reported to lead to cholesterol depletion and to inhibit tBid-induced Cytochrome $c$ release, leading the authors to propose that cholesterol-rich MOM microdomains might be essential for MOM permeabilization. ${ }^{30}$ Unpublished results from our group show that at this concentration $\mathrm{M} \beta \mathrm{c}$ prevents $\mathrm{tBid}$-induced MOM permeabilization, but does not modify the cholesterol content of HeLa mitochondria, suggesting that the effect of $\mathrm{M} \beta \mathrm{c}$ on $\mathrm{MOM}$ permeabilization might not be due to cholesterol depletion.

During apoptosis, activated Bax does not spread evenly on the whole surface of mitochondria but displays a punctuate distribution. These foci were reported to coincide with proteins of the mitochondrial fission machinery. ${ }^{31}$ This could indicate that Bax needs to cooperate with fission proteins to permeabilize MOMs. An alternative, but not mutually exclusive possibility, is that it reflects the specific association of oligomerized Bax with some lipid microdomains, and/or its exclusion from others. Our results suggest that exclusion of Bax from ordered MOM regions is important for its proper activation. The existence of lipid microdomains might also be important for mitochondrial fission. Mitochondrial fission requires local bending of lipid bilayers, which appears to be disfavoured in ordered membrane microdomains. ${ }^{32}$

These in vitro observations prompted us to test whether increasing cholesterol levels in mitochondria would also alter Bax activation in vivo and consequently retard cell death. We used U18666A as a tool to increase mitochondrial cholesterol levels and to test the validity of our in vitro results in a more physiological system. We showed in HeLa cells that U18666A promotes a rise in the mitochondrial cholesterol content. This enrichment was associated with a reduced capacity of isolated mitochondria to sustain tBid-induced Bax oligomerization and MOM permeabilization, thereby validating data obtained with liposomes. A similar delay in Bax activation and cell death was observed in U18666A-treated cells exposed to Bax/Bak-dependent apoptotic stimuli. The beneficial antiapoptotic effect provided by increased mitochondrial cholesterol levels was probably minimized by concomitant toxic sideeffects exerted by U18666A. ${ }^{27,33}$ This toxicity was revealed in a model of Bax/Bak-independent apoptosis as the compound was found to accelerate the death of 293T cells exposed to FasL. Therefore, although we present U18666A as a suitable tool to modulate mitochondrial cholesterol levels and to delay Bax activation and MOM permeabilization, its antiapoptotic properties are limited due to its toxic effects.

Interestingly, many tumour cells were reported to have high levels of cholesterol in their mitochondrial membranes. ${ }^{34-37}$ The accumulation of cholesterol in the mitochondria of tumour cells was proposed to increase their resistance to large amplitude swelling, ${ }^{38}$ decrease proton leak across the mitochondrial inner membrane, ${ }^{34}$ and to modify the activities of several transmembrane proteins. ${ }^{37,38}$ We have now shown that high amounts of cholesterol in mitochondrial membranes can also 
inhibit Bax activation in a tumour cell line (HeLa cells), and increase its resistance to stress-induced apoptosis. This mechanism could contribute at least in part to the proapoptotic activity of statins, which are inhibitors of the ratelimiting enzyme of the mevalonate pathway involved in the synthesis of cholesterol, and which are under clinical trial as chemotherapeutic agents. ${ }^{39}$

Previous studies suggested that lipids modulate the process of MOM permeabilization; our in vitro and in vivo experiments now reveal that Bax oligomerization is tightly controlled by the physical properties of bilayers. MOM permeabilization is crucial for the completion of many apoptotic programmes, and it is becoming clear that its comprehension will require a better understanding of the process, integrating both protein and lipid partners. The fact that many cancer cells have increased mitochondrial cholesterol levels could be associated with an increased resistance towards Bax activation, and understanding how mitochondrial cholesterol levels are regulated, could allow the discovery of new routes to impact on their survival.

\section{Materials and Methods}

Recombinant proteins. Recombinant His-Bax, caspase-8-cleaved Bid and Bidmlll-2 (192A, G93A, D94A and E95A) and His-Bcl-x L lacking its C-terminal domain were purified as described previously. ${ }^{21,40,41}$ His-tagged recombinant human Fis was cloned in pTYB1 vector (New England Biolabs) with a C-terminal intein-chitin ending, and expressed in Escherichia coli. Cells were lysed in the presence of 1\% deoxycholic acid and 1\% IGEPAL, and broken mechanically with a French press (SLM Instruments Inc.). After centrifugation, the bacterial extracts were loaded on a Nickel-nitriloacetic acid-agarose column, washed with $25 \mathrm{mM} \mathrm{Hepes} \mathrm{pH} \mathrm{8,500} \mathrm{mM}$ $\mathrm{NaCl}, 0.5 \%$ IGEPAL, and eluted with imidazole $(200 \mathrm{mM})$. Fis-containing fractions were then loaded on a column filled with chitin beads (New England Biolabs), and eluted in the presence of $50 \mathrm{mM}$ DTT to cleave the intein-chitin tag. Fis was then dialysed against $25 \mathrm{mM}$ Hepes $\mathrm{pH} 7.5,1 \%$ CHAPS, $0.5 \mathrm{M} \mathrm{NaCl}$ and $5 \mathrm{mM}$ $\beta$-mercaptoethanol, and aliquots were stored at $-80^{\circ} \mathrm{C}$.

Cell culture and apoptosis induction. HeLa cells (European Collection for Cell Cultures) and 293T cells were grown in DME supplemented with $10 \%$ FCS, $100 \mathrm{u} / \mathrm{ml}$ penicillin, $0.1 \mathrm{mg} / \mathrm{ml}$ streptomycin, $2 \mathrm{mM}$ glutamine, and maintained in $5 \% \mathrm{CO}_{2}$ at $37^{\circ} \mathrm{C}$. Cell culture reagents were from Sigma. Twenty-four hours after plating, U18666A (3- $\beta$-[2-(diethylamino)ethoxy]androst-5-en-17-one, $3 \mu \mathrm{g} / \mathrm{ml}$ in water, Biomol) was added to the medium and left for $48 \mathrm{~h}$ before experiments were undertaken. Apoptosis was induced with UV using a UV Stratalinker 2400 apparatus (Stratagene), with actinomycin D ( $3 \mu \mathrm{M}$, Sigma), with staurosporine $(1 \mu \mathrm{M}$, Sigma), or with FasL $(50 \mathrm{ng} / \mathrm{ml}, \mathrm{R} \& \mathrm{D}$ Systems) and cycloheximide $(10 \mu \mathrm{M}$, Sigma)

Cell fractionation and mitochondria isolation. Crude mitochondria and cytosolic extracts were prepared from HeLa cells using a protocol similar to the one described in Parone et al. ${ }^{42}$ In brief, cells were mechanically broken three times using a $2 \mathrm{ml}$ glass/glass Dounce homogenizer (Kontes) (20, 15, and 15 strokes). Homogenates were cleared at $1500 \mathrm{~g}$ and mitochondria were spun down at $10000 \mathrm{~g}$. Protein concentrations were measured with a Bradford assay (Bio-Rad Laboratories). Proteins in the supernatants (cytosolic fractions) were precipitated with TCA $(10 \%, 30 \mathrm{~min}$ on ice). Samples were either directly analyzed by immunoblotting or mitochondria were processed for further tests.

Immunoblotting and immunocytochemistry. Samples were separated in polyacrylamide gels and transferred to Hybond-P membranes (Amersham Pharmacia). Immunoblotting was performed with the following antibodies: Bax (BD-Pharmingen), tBid (R\&D Systems), Fis and Caspase-3 (Alexis Biochemicals), polyclonal Cytochrome c, Smac/DIABLO (ProSci Inc.), Bcl-- $\mathrm{L}_{\mathrm{L}}$ (Transduction Laboratories), Bak (Upstate Biotechnology), polyclonal SLP-2 (Da Cruz S, in preparation), VDAC (Santa Cruz Biotechnology), Tom22 and Catalase (Sigma), Aldolase (Chemicon), Calnexin and Lamp1 (Affinity Bioreagents), SKL (Zymed Laboratories), Rab5 (gift from Reinhard Jahn), GS28 (StressGen
Biotechnologies Corp.). Secondary antibodies (antimouse and antirabbit from DakoCytomation, antigoat from Santa Cruz Biotechnology) were coupled to horseradish peroxidase and signals were detected by ECL. For quantifications, images were acquired using a CCD camera-based system (GeneGnome, Syngene) and band intensities were quantified using ImageJ. They were then normalized with the intensities of the bands associated with SLP-2, and U18666A-treated/untreated ratios were calculated. Statistical analysis was performed with a paired Student's t-test.

Immunocytochemistry with a monoclonal Cytochrome $c$ antibody (BD-Pharmingen) was performed essentially as described previously. ${ }^{42}$

Liposome preparation and in vitro Bax activation assay. Egg yolk $\mathrm{PC}$, bovine heart $\mathrm{CL}$, bovine brain $\mathrm{PE}$, bovine liver PI, cholesterol, DSPC and DMPC were from Sigma; DOPC was from Avanti Polar Lipids. Lipids were mixed (w/w ratios) and dried under nitrogen at $30^{\circ} \mathrm{C}$. They were then incubated with $\mathrm{KCl}$ buffer (125 mM KCl, $4 \mathrm{mM} \mathrm{MgCl}, 5 \mathrm{mM} \mathrm{KH}_{2} \mathrm{PO}_{4}, 10 \mathrm{mM}$ Hepes pH 7.4) for 20 min on a rotating wheel, and completely resuspended by gentle vortexing. Five cycles of freezing in liquid nitrogen and thawing at $30^{\circ} \mathrm{C}$ were performed. Unless otherwise stated, liposomes (500 $\mu \mathrm{g}$ per test, $1 \mu \mathrm{g} / \mu \mathrm{l}$ in $\mathrm{KCl}$ buffer) were incubated with recombinant His-Bax $(100 \mathrm{nM}) \pm \mathrm{tBid}(10 \mathrm{nM})$ for $30 \mathrm{~min}$ at $30^{\circ} \mathrm{C}$. Unbound proteins were removed by ultracentrifugation $\left(30 \mathrm{~min}, 180000 \mathrm{~g}, 10^{\circ} \mathrm{C}\right)$. The pellets were then resuspended in $\mathrm{KCl}$ buffer; half of each pellet was kept to analyze the binding of Bax and tBid, while the other half was incubated with trypsin $(0.17 \mathrm{mg} / \mathrm{ml})$ for $2 \mathrm{~h}$ at $30^{\circ} \mathrm{C}$. Reactions were stopped with trypsin inhibitor $(1.4 \mathrm{mg} / \mathrm{ml}$, Sigma). Proteins bound to the liposomes and resistant to trypsin digestion were analyzed by immunoblotting. To test the insertion of Bax in lipid bilayers, the pellets were instead incubated with sodium carbonate $(0.1 \mathrm{M})$ for $30 \mathrm{~min}$ on ice. After an ultracentrifugation, pellets were analyzed by immunoblotting.

Steady-state fluorescence anisotropy. Liposomes were prepared as described previously. DPH ( $5 \mu \mathrm{M}$, Sigma) was added to the mixtures, and a 30-min incubation was carried out at $30^{\circ} \mathrm{C}$ in the dark. Samples were analyzed at RT in a Cary Eclipse Fuorimeter, scanning excitation wavelengths between 300 and $415 \mathrm{~nm}$, and using an emission wavelength of $425 \mathrm{~nm}$. Similarly to Aleardi et al., ${ }^{22}$ fluorescence anisotropy was calculated from the intensities of the light emitted parallel or perpendicular to the polarization plane of the incident light, but the anisotropy was averaged between excitation wavelengths of 300 and $360 \mathrm{~nm}$. Statistical significance was assessed with a paired Student's $t$-test.

Size exclusion chromatography. Unless otherwise stated, liposomes were incubated with recombinant His-Bax $(100 \mathrm{nM})$ and tBid $(10 \mathrm{nM})$ for $30 \mathrm{~min}$ at $30^{\circ} \mathrm{C}$. CHAPS was added to a final concentration of $2 \%$, and a $1 \mathrm{~h}$ incubation was carried out on ice. Insoluble material was removed by centrifugation ( $30 \mathrm{~min}$, $100000 \mathrm{~g}, 4^{\circ} \mathrm{C}$ ), and the supernatant was loaded on a sephacryl S200 column (Amersham Pharmacia). A $130 \mathrm{ml}$ run in $25 \mathrm{mM}$ Hepes pH 7.5, 1\% CHAPS, $2 \mathrm{mM}$ DTT was performed and $2 \mathrm{ml}$ fractions were recovered. The distribution of Bax was analyzed by immunoblotting.

FD20 release assays. FD20 $(20 \mathrm{mg} / \mathrm{ml}$, Sigma) was entrapped in liposomes $(100 \mu \mathrm{g} / \mu \mathrm{l})$, and five cycles of freezing in liquid nitrogen and thawing at $30^{\circ} \mathrm{C}$ were performed. Liposomes were diluted in $\mathrm{KCl}$ buffer, and left overnight to sediment. The supernatants were discarded and liposomes were resuspended in $\mathrm{KCl}$ buffer. Bax activation assays were performed as described above and the fluorescence of the supernatants recovered after the ultracentrifugation step was monitored using a Chameleon plate reader (Hidex). The fraction of FD20 released in supernatants relative to maximal release obtained with $1 \%$ Triton was calculated. Statistical significance was assessed with a paired Student's t-test.

Lipid extraction and thin-layer chromatography analysis. Lipids were extracted from isolated crude mitochondria: 2.6 vol $\mathrm{MeOH}$ and $1.3 \mathrm{vol} \mathrm{CHCl}_{3}$ were added, samples were thoroughly vortexed and incubated on ice for $10 \mathrm{~min}$. $\mathrm{CHCl}_{3}(1.3 \mathrm{vol})$ and water $(1.3 \mathrm{vol})$ were further added, and samples were vortexed and incubated on ice for $10 \mathrm{~min}$. They were then centrifuged $\left(5 \mathrm{~min}, 250 \mathrm{~g}, 4^{\circ} \mathrm{C}\right)$ and the bottom organic phase was recovered and dried under nitrogen at $30^{\circ} \mathrm{C}$. Dried lipids were then resuspended in a small volume of $\mathrm{CHCl}_{3}$ and lipids corresponding to $20 \mu \mathrm{g}$ (for cholesterol) or $50 \mu \mathrm{g}$ (for the other lipids) of proteins were spotted on silica gel 60 TLC plates (Merck). Cholesterol was developed with a heptane/ether/ acetic acid (18/6/2) mixture, while other phospholipids were developed with a 
$\mathrm{CHCl}_{3} / \mathrm{MeOH} /$ water (43/17/2.7) mixture. Plates were air-dried, bathed in a copper (II) acetate solution (3\%) in $8 \% \mathrm{H}_{3} \mathrm{PO}_{4}$ and placed for $5-15 \mathrm{~min}$ in an oven at $120^{\circ} \mathrm{C}$. Spot intensities were quantified using ImageJ (http://rsb.info.nih.gov/ij/). Commercial lipids were used to identify the spots and to establish standard curves, choosing the conditions as to be in the linear range of response for individual lipids. Statistical significance was assessed with a paired Student's $t$-test.

Mitochondrial cholesterol content. The total cholesterol content of $10 \mu \mathrm{g}$ of isolated crude mitochondria was determined with the Amplex Red Cholesterol Assay Kit from Invitrogen, following the instructions of the manufacturer. Statistical significance was assessed with a paired Student's $t$-test.

Mitochondria fractionation on a sucrose gradient. Equal amounts of mitochondria isolated from control or U18666A-treated cells were covered with $4 \mathrm{ml}$ of a $48-8 \%$ continuous sucrose gradient (in $10 \mathrm{mM}$ Hepes pH 7.4, $1 \mathrm{mM}$ EDTA). They were then ultracentrifuged $\left(16 \mathrm{~h}, 100000 \mathrm{~g}, 4^{\circ} \mathrm{C}\right)$, and $500 \mu \mathrm{l}$ fractions were collected from the top of the gradients. Protein concentrations were determined as above and protein-containing fractions were analyzed by immunoblotting.

tBid-induced Bax activation in isolated mitochondria. Crude mitochondria were resuspended at $1 \mu \mathrm{g} / \mu \mathrm{l}$ in $\mathrm{KCl}$ buffer supplemented with succinate $(5 \mathrm{mM})$ and EGTA $(0.5 \mathrm{mM})$. tBid $(20 \mathrm{nM})$ was added to the samples and incubations were carried out at RT. At the indicated time points, samples were centrifuged $\left(5 \mathrm{~min}, 10000 \mathrm{~g}, 4^{\circ} \mathrm{C}\right)$; supernatants were recovered and analyzed by immunoblotting, and pellets were processed further. To assess Bax and tBid insertion, loosely associated proteins were detached from membranes by alkaline extraction, the pellets were resuspended at $0.5 \mu \mathrm{g} / \mu \mathrm{l}$ in freshly made $\mathrm{Na}_{2} \mathrm{CO}_{3}$ $(0.1 \mathrm{M})$; they were then incubated on ice for $30 \mathrm{~min}$ and spun $30 \mathrm{~min}$ at $100000 \mathrm{~g}$, $4^{\circ} \mathrm{C}$. These pellets were analyzed by immunoblotting. To assess Bax oligomerization, pellets were washed once in $\mathrm{KCl}$ buffer and spun $5 \mathrm{~min}$ at $10000 \mathrm{~g}$; pellets were then resuspended at $4 \mu \mathrm{g} / \mu \mathrm{l}$ in $\mathrm{KCl}$ buffer, trypsin was added $(0.17 \mathrm{mg} / \mathrm{ml})$ and $1 \mathrm{~h} 30$ digestions were carried out at $30^{\circ} \mathrm{C}$. Samples were analyzed by immunoblotting.

Acknowledgements. We thank Professor Jean Gruenberg (University of Geneva) for his advice, help and encouragements during the course of this work, and for providing us with anti-Lamp1 antibodies. We thank Professor Eric Vauthey (University of Geneva), Dr Bernhard Lang and Alexandre Fürstenberg for help and guidance with the fluorescence anisotropy technique. We thank Professor Christoph Borner (University of Freiburg) for providing us with anti-Bak antibodies, Professor Jürg Tschopp (University of Lausanne) for the 293T cells and Professor Reinhard Jahn (Max-Planck Institute for Biophysical Chemistry, Goettingen) for anti-Rab5 antibodies. We express our deepest gratitude to Professor Edwin Lucken and $\mathrm{Dr}$ Julien Häsler for critical comments about the manuscript. This work was funded by the Swiss National Science Foundation (subsidy 3100A0-109419/1), OncoSuisse Trust, and the Geneva Department of Education.

1. Lucken-Ardjomande S, Martinou JC. Newcomers in the process of mitochondria permeabilization. J Cell Sci 2005; 118: 473-483.

2. Antonsson B, Montessuit S, Sanchez B, Martinou JC. Bax is present as a high molecular weight oligomer/complex in the mitochondrial membrane of apoptotic cells. J Biol Chem 2001; 276: 11615-11623.

3. Wei MC, Lindsten T, Mootha VK, Weiler S, Gross A, Ashiya M et al. tBID, a membranetargeted death ligand, oligomerizes BAK to release cytochrome $c$. Genes Dev 2000; 14: 2060-2071.

4. Eskes $\mathrm{R}$, Desagher $\mathrm{S}$, Antonsson $\mathrm{B}$, Martinou JC. Bid induces the oligomerization and insertion of Bax into the outer mitochondrial membrane. Mol Cell Biol 2000; 20: 929-935.

5. Upton JP, Valentijn AJ, Zhang L, Gilmore AP. The N-terminal conformation of Bax regulates cell commitment to apoptosis. Cell Death Differ 2007; 14: 932-942.

6. Borner $\mathrm{C}$. The $\mathrm{Bcl}-2$ protein family: sensors and checkpoints for life-or-death decisions. Mol Immunol 2003; 39: 615-647.

7. Galonek HL, Hardwick JM. Upgrading the BCL-2 network. Nat Cell Biol 2006; 8: 1317-1319.

8. Lucken-Ardjomande S, Martinou JC. Regulation of Bcl-2 proteins and of the permeability of the outer mitochondrial membrane. C R Biol 2005; 328: 616-631.

9. Kuwana T, Mackey MR, Perkins G, Ellisman MH, Latterich M, Schneiter R et al. Bid, Bax, and lipids cooperate to form supramolecular openings in the outer mitochondrial membrane. Cell 2002; 111: 331-342.
10. Terrones $\mathrm{O}$, Antonsson B, Yamaguchi $\mathrm{H}$, Wang HG, Liu Y, Lee RM et al. Lipidic pore formation by the concerted action of pro-apoptotic BAX and tBID. J Biol Chem 2004; 279 30081-30091.

11. Roucou X, Montessuit S, Antonsson B, Martinou JC. Bax oligomerization in mitochondrial membranes requires tBid (caspase-8-cleaved Bid) and a mitochondrial protein. Biochem $J$ 2002; 368: 915-921.

12. Booth PJ. Sane in the membrane: designing systems to modulate membrane proteins. Curr Opin Struct Biol 2005; 15: 435-440.

13. Gonzalvez F, Gottlieb E. Cardiolipin: setting the beat of apoptosis. Apoptosis 2007; 12 877-885.

14. Esposti MD, Cristea IM, Gaskell SJ, Nakao Y, Dive C. Proapoptotic Bid binds to monolysocardiolipin, a new molecular connection between mitochondrial membranes and cell death. Cell Death Differ 2003; 10: 1300-1309.

15. Kagan VE, Tyurin VA, Jiang J, Tyurina YY, Ritov VB, Amoscato AA et al. Cytochrome acts as a cardiolipin oxygenase required for release of proapoptotic factors. Nat Chem Biol 2005; 1: 223-232.

16. Sandra F, Degli Esposti M, Ndebele K, Gona P, Knight D, Rosenquist M et al. Tumor necrosis factor-related apoptosis-inducing ligand alters mitochondrial membrane lipids. Cancer Res 2005; 65: 8286-8297.

17. Epand RF, Martinou JC, Montessuit S, Epand RM. Fatty acids enhance membrane permeabilization by pro-apoptotic Bax. Biochem J 2004; 377: 509-516.

18. Goping IS, Gross A, Lavoie JN, Nguyen M, Jemmerson R, Roth K et al. Regulated targeting of BAX to mitochondria. J Cell Biol 1998; 143: 207-215.

19. Antonsson B, Montessuit S, Lauper S, Eskes R, Martinou JC. Bax oligomerization is required for channel-forming activity in liposomes and to trigger cytochrome $c$ release from mitochondria. Biochem J 2000; 345 (Part 2): 271-278.

20. Sasahara K, Yagi H, Naiki H, Goto Y. Heat-induced conversion of beta(2)-microglobulin and hen egg-white lysozyme into amyloid fibrils. J Mol Biol 2007; 372: 981-991.

21. Desagher S, Osen-Sand A, Nichols A, Eskes R, Montessuit S, Lauper S et al. Bid-induced conformational change of $\mathrm{Bax}$ is responsible for mitochondrial cytochrome $c$ release during apoptosis. J Cell Biol 1999; 144: 891-901.

22. Aleardi AM, Benard G, Augereau O, Malgat M, Talbot JC, Mazat JP et al. Gradual alteration of mitochondrial structure and function by beta-amyloids: importance of membrane viscosity changes, energy deprivation, reactive oxygen species production, and cytochrome c release. J Bioenerg Biomembr 2005; 37: 207-225.

23. Lange $Y, Y$, J, Rigney M, Steck T. Cholesterol movement in Niemann-Pick type $C$ cells and in cells treated with amphiphiles. J Biol Chem 2000; 275: 17468-17475.

24. Liscum L, Faust JR. The intracellular transport of low density lipoprotein-derived cholestero is inhibited in Chinese hamster ovary cells cultured with 3-beta-[2-(diethylamino) ethoxy]androst-5-en-17-one. J Biol Chem 1989; 264: 11796-11806.

25. Sexton RC, Panini SR, Azran F, Rudney H. Effects of 3 beta-[2-(diethylamino) ethoxy]androst-5-en-17-one on the synthesis of cholesterol and ubiquinone in rat intestinal epithelial cell cultures. Biochemistry 1983; 22: 5687-5692.

26. Yu W, Gong JS, Ko M, Garver WS, Yanagisawa K, Michikawa M. Altered cholesterol metabolism in Niemann-Pick type $\mathrm{C} 1$ mouse brains affects mitochondrial function. J Bio Chem 2005; 280: 11731-11739.

27. Cenedella RJ, Sexton PS, Krishnan K, Covey DF. Comparison of effects of U18666A and enantiomeric U18666A on sterol synthesis and induction of apoptosis. Lipids 2005; 40 635-640.

28. Annis MG, Soucie EL, Dlugosz PJ, Cruz-Aguado JA, Penn LZ, Leber B et al. Bax forms multispanning monomers that oligomerize to permeabilize membranes during apoptosis. EMBO J 2005; 24: 2096-2103.

29. Daum G. Lipids of mitochondria. Biochim Biophys Acta 1985; 822: 1-42.

30. Garofalo T, Giammarioli AM, Misasi R, Tinari A, Manganelli V, Gambardella L et al. Lipid microdomains contribute to apoptosis-associated modifications of mitochondria in $\mathrm{T}$ cells. Cell Death Differ 2005; 12: 1378-1389.

31. Youle RJ, Karbowski M. Mitochondrial fission in apoptosis. Nat Rev Mol Cell Biol 2005; 6 : 657-663.

32. Roux A, Cuvelier D, Nassoy P, Prost J, Bassereau P, Goud B. Role of curvature and phase transition in lipid sorting and fission of membrane tubules. EMBO J 2005; 24 $1537-1545$

33. Koh CH, Whiteman M, Li QX, Halliwell B, Jenner AM, Wong BS et al. Chronic exposure to U18666A is associated with oxidative stress in cultured murine cortical neurons. $J$ Neurochem 2006; 98: 1278-1289.

34. Baggetto LG, Clottes E, Vial C. Low mitochondrial proton leak due to high membrane cholesterol content and cytosolic creatine kinase as two features of the deviant bioenergetics of Ehrlich and AS30-D tumor cells. Cancer Res 1992; 52 4935-4941.

35. Crain RC, Clark RW, Harvey BE. Role of lipid transfer proteins in the abnormal lipid content of Morris hepatoma mitochondria and microsomes. Cancer Res 1983; 43 3197-3202.

36. Feo F, Canuto RA, Bertone G, Garcea R, Pani P. Cholesterol and phospholipid composition of mitochondria and microsomes isolated from morris hepatoma 5123 and rat liver. FEBS Lett 1973; 33: 229-232.

37. Parlo RA, Coleman PS. Enhanced rate of citrate export from cholesterol-rich hepatoma mitochondria. The truncated Krebs cycle and other metabolic ramifications of mitochondrial membrane cholesterol. J Biol Chem 1984; 259: 9997-10003. 
38. Graham JM, Green C. The properties of mitochondria enriched in vitro with cholesterol. Eur J Biochem 1970; 12: 58-66.

39. Wong WW, Dimitroulakos J, Minden MD, Penn LZ. HMG-CoA reductase inhibitors and the malignant cell: the statin family of drugs as triggers of tumor-specific apoptosis. Leukemia 2002; 16: 508-519.

40. Kudla G, Montessuit S, Eskes R, Berrier C, Martinou JC, Ghazi A et al. The destabilization of lipid membranes induced by the $\mathrm{C}$-terminal fragment of caspase 8-cleaved bid is inhibited by the N-terminal fragment. J Biol Chem 2000; 275 : 22713-22718.

41. Montessuit S, Mazzei G, Magnenat E, Antonsson B. Expression and purification of fulllength human Bax alpha. Protein Expr Purif 1999; 15: 202-206.

42. Parone PA, James DI, Da Cruz S, Mattenberger Y, Donze O, Barja F et al. Inhibiting the mitochondrial fission machinery does not prevent Bax/Bak-dependent apoptosis. Mol Cell Biol 2006; 26: 7397-7408.

Supplementary Information accompanies the paper on Cell Death and Differentiation website (http://www.nature.com/cdd) 